\title{
DECISION SUPPORT SYSTEM IN DETERMINING THE BEST JUDO ATHLETE USING AHP METHOD
}

\author{
Dinar Ajeng Kristiyanti'; Garth Wishnuwardhana Pangemanan² \\ Informatics Engineering ${ }^{1}$, Information System ${ }^{2}$ \\ STMIK Nusa Mandiri ${ }^{1,2}$ \\ http://www.nusamandiri.ac.id \\ dinar@nusamandiri.ac.id ${ }^{1}$, garth.pangemanan@gmail.com²
}

\begin{abstract}
To determine the best Judo athlete, Rajawali Judo Club of Battalion Arhanud 1 Divif 1 Kostrad has several obstacles such as making a decision in determining the best Judo athlete by the Coach and the Achievement Development which only based on experience which is estimated without the existence of any system. This results in subjectivity and the absence of a strong basis for competent objective decision making which then triggers gaps between athletes. The absence of specific criteria creates that result in not targeting the selection of the best Judo athletes. For this reason, a method in this case is needed to choose the AHP (Analytical Hierarchy Process) method and a number of criteria as indicators in determining the best Judo athlete. While the referenced criteria are Self-Dropping Technique (Ukemi), Slamming Technique (Nagewaza), Lockdown or Lying Technique (Katamewaza), Discipline and Achievement. The purpose of this study is expected to produce statistical data as an evaluation material for the training team to minimize or even eliminate the gap between fellow Judo athletes at the Rajawali Judo Club of Battalion Arhanud 1 Divif 1 Kostrad. The result of this study is based on Analytical Hierarchy Process calculations, obtained the most important priority criteria in determining the best Judo athlete in which the Achievement criteria with value 0.325 or equivalent to $32 \%$, then followed by Disciplinary criteria with value 0.227 or equivalent to $23 \%$, Slamming Technique criteria (Nage-waza) with value 0.211 or equivalent to 21\%, Lockdown/Laying Technique criteria (Katame-waza) with value 0.125 or equivalent to $12 \%$ and in the last rank the SelfDropping Technique criteria (Ukemi) with value 0.112 or equivalent to $11 \%$.
\end{abstract}

Keyword: Judo, Decision Support System, AHP.

Intisari- Untuk menentukan atlet Judo terbaik, Rajawali Judo Club Batalyon Arhanud 1 Divif 1 Kostrad memiliki beberapa kendala seperti pengambilan suatu keputusan dalam penentuan atlet Judo terbaik oleh Pelatih maupun bagian Pinpres (Pembinaan Prestasi) yaitu hanya berdasarkan pengalaman yang sifatnya perkiraan tanpa adanya alat bantu berupa sistem apapun. Hal ini menimbulkan subyektivitas dan tidak adanya dasar kuat pengambilan keputusan objektif yang kompeten yang lalu memicu terjadinya kesenjangan di antara sesama atlet. Tidak adanya kriteria khusus yang mengakibatkan tidak tepat sasaran pada penyeleksian atlet Judo terbaik. Untuk itu dibutuhkan sebuah metode dalam hal ini dipilih dipilih metode AHP (Analytical Hierarchy Process) dan sejumlah kriteria sebagai indikator dalam menentukan atlet Judo terbaik maka. Adapun kriteria yang menjadi acuan adalah Teknik Menjatuhkan Diri (Ukemi), Teknik Bantingan (Nage-waza), Teknik Kuncian atau Berbaring (Katame-waza), Kedisiplinan dan Prestasi. Tujuan penelitian ini diharapkan dapat menghasilkan data statistik sebagai bahan evaluasi bagi tim pelatih, sehingga dapat meminimalisir atau bahkan menghilangkan kesenjangan diantara sesama atlet Judo pada Rajawali Judo Club Batalyon Arhanud 1 Divif 1 Kostrad. Hasil penelitian ini adalah berdasarkan perhitungan Analytical Hierarchy Process, diperoleh prioritas kriteria yang paling penting dalam menentukan atlet Judo terbaik dimana kriteria Prestasi dengan bobot 0,325 atau setara dengan $32 \%$, lalu selanjutnya diikuti oleh kriteria Kedisplinan dengan bobot 0,227 atau setara dengan 23\%, kriteria Teknik Bantingan (Nagewaza) dengan bobot 0,211 atau setara dengan 21\%, Kriteria Teknik Kuncian / Berbaring (Katame-waza) dengan bobot 0,125 atau setara dengan $12 \%$ dan di peringkat terakhir yaitu kriteria Teknik Menjatuhkan Diri (Ukemi) dengan bobot 0,112 atau setara dengan $11 \%$.

Kata Kunci: Judo, Sistem Pendukung Keputusan, AHP.

\section{INTRODUCTION}

According to Ben Haryo in (Sundari, 2018) "Judo is a martial art, sport and philosophy rooted in Japan. Judo was developed from an ancient Japanese martial art called Jujutsu. Jujutsu which is an art of defense and attack using bare hands or 
short weapons, was developed into Judo by Kano Jigoro in 1882. This sport became a model of Japanese martial arts, gendai budo developed from an old school (koryu)". Judo as a competitive martial sport both at national and international level requires a reliable system in the selection of sustainable junior athletes, in this case specifically the application of a Decision Support System to determine the best Judo athletes.

In addition, Turban in (Agung \& Ricky, 2016) states that Decision Support System or DSS is a system intended to support managerial decision makers in a semitructured decision situation. DSS is intended to be a tool for decision makers to expand their capabilities, but not to replace their judgment. DSS is intended for decisions that require judgment or decisions that cannot be supported by algorithms at all. In the Decision Support System (SPK) there are a number of supporting methods including SAW (Simple Additive Weighting) (Ruskan et al., 2013), TOPSIS (Technique for Order by Similarity to Ideal Solution) (Desi Ratna et al., 2018) (Herman Firdaus et al., 2016) (Mallu, 2015) (Agung \& Ricky, 2016), WP (Weighted Product), PROMETHEE (Imandasari et al., 2018), Profile Matching (Kristiyanti, 2017), and AHP (Analytic Hierarchy Process) (Saefudin \& Wahyuningsih, 2014) (Polmetra et al., 2015) (Herman Firdaus et al., 2016) (Mardiati \& Oktafianto, 2017) (Nurdiyanto \& Meilia, 2016)(Kurniawan \& Gusrianty, 2018) (Handayani \& Marzuki, 2018) (Desi Ratna et al., 2018) (Nadeak et al., 2018). While the type of method chosen as a suitable method refers to a number of previous similar studies that are often used to determine the best athlete selection process is the AHP method

Initially the method of AHP (Analytical Hierarchy Process) was developed by Thomas L. Saaty. According to (Mardiati \& Oktafianto, 2017) "Hierarchy is defined as a representation of a complex problem in a multi-level structure where the first level is the goal, followed by the level of factors, criteria, sub-criteria, and so on up to the last level of alternatives. With hierarchy, a complex problem can be broken down into groups which are then organized into a hierarchical form so that the problem will appear more structured and systematics". According to (Nurdiyanto \& Meilia, 2016) "a number of advantages of the AHP method are the hierarchical structure as a consequence of the criteria chosen to the deepest sub-criteria, taking into account validity to the limit of inconcentration tolerance as criteria and alternatives chosen by decision makers and take into account the resilience or resilience of the output of sensitivity analysis of decision making".
Rajawali Judo Club of Battalion Arhanud 1 Divif 1 Kostrad which focuses on fostering student athletes and students to excel in regional competitions such as PORDA (Regional Sports Week), PORPROV (Provincial Sports Week) and national events such as the Judo National Championship and PON (National Sports Week). The obstacle faced by Rajawali Judo Club so far is the unavailability of a computerized system for supporting decision making. This shows that, making a decision in the determination of the best Judo athletes by the Coach and the Pinpres (Achievement Development) is only based on the experience of an estimated nature without the existence of tools in the form of any system. This results in subjectivity and the absence of a strong basis for competent objective decision making which then triggers gaps between fellow athletes. The absence of specific criteria that result in not targeting the selection of the best Judo athletes

In an effort to obtain superior juniors of Judo athletes, Rajawali Judo Club Battalion Arhanud 1 Divif 1 Kostrad certainly must have a number of clear criteria in selecting the best Judo athlete candidates, the selection process is very influential to be able to attract potential athletes who have great potential. Reflecting on the above problem, the author considers the need for a system that can help in making a decision. Coach and Pinpres (Achievement Development) based on existing criteria and value determined by proposing a solution that is making a Decision Support System in Determining the Best Judo Athletes Using the AHP Method in Rajawali Judo Club Battalion Arhanud 1 Divif 1 Kostrad

\section{MATERIAL AND METHOD}

\section{A. Decision Support System}

"Decision Support Systems (DSS) are usually built to support solutions to a problem or to an opportunity" (Sanyoto et al., 2017). "DSS aims to provide information, guide, and predict for information users so they can make better decisions" (Ariani, 2017).

\section{B. Analytical Hierarchy Process (AHP) Method}

"AHP (Analytical Hierarchy Process) method is a method of comprehensive decision making. This method takes into account for both qualitative and quantitative matters at once" (Stevanus et al., 2017). (Nadeak et al., 2018) suggested that "Analytical Hierarchy Process is a concept for multi criteria based decision making. Some criteria are compared with one another (level of importance). AHP becomes a method of determining or making decisions that combines the principles of 
subjectivity and objectivity of the DSS maker or his decision ".

\section{RESULTS AND DISCUSSION}

\section{A. Hierarchical Structure}

The hierarchical structure in the selection of the best Judo athletes uses the Analytical Hierarchy Process (AHP) method, in Figure 1 below:

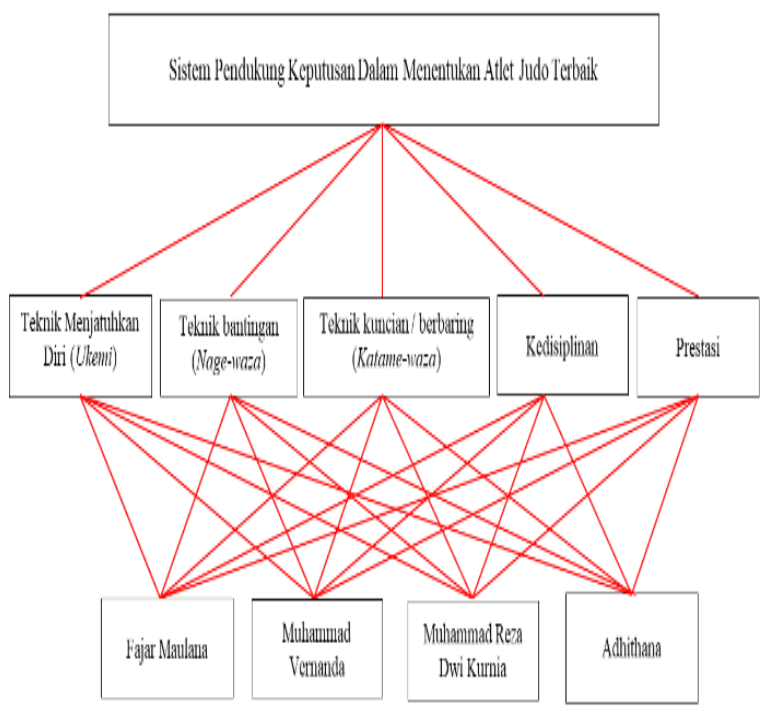

Source: (Kristiyanti \& Pangemanan, 2020)

Figure 1. Hierarchical Structure in Determining the Best Judo Athletes

\section{B. Data Culculation Using AHP Method}

After getting the data from the questionnaire result then the data is calculated using the Analytical Hierarchy Process (AHP) method).

\section{Based on Criteria}

Comparasion matrix in pairs based on criteria from the processed questionnaire data result in the following table 1 :

Table 1. Matrix Based on Criteria

\section{Criteria of Comparation Matrix in Pairs}

\begin{tabular}{lccccc}
\hline \multicolumn{1}{c}{ Criteria } & Ukemi & $\begin{array}{c}\text { Nage- } \\
\text { waza }\end{array}$ & $\begin{array}{c}\text { Katame- } \\
\text { waza }\end{array}$ & $\begin{array}{c}\text { Discip } \\
\text { linary }\end{array}$ & Achievement \\
\hline Ukemi & 1.000 & 0.382 & 0.794 & 0.550 & 0.464 \\
\hline $\begin{array}{l}\text { Nage- } \\
\text { waza }\end{array}$ & 2.621 & 1.000 & 2.080 & 0.693 & 0.481 \\
\hline $\begin{array}{l}\text { Katame- } \\
\text { waza }\end{array}$ & 1.260 & 0.481 & 1.000 & 0.481 & 0.481 \\
\hline $\begin{array}{l}\text { Disciplina } \\
\text { ry }\end{array}$ & 1.817 & 1.442 & 2.080 & 1.000 & 0.550 \\
\hline $\begin{array}{l}\text { Achievem } \\
\text { ent }\end{array}$ & 2.154 & 2.080 & 2.080 & 1.817 & 1.000 \\
\hline $\begin{array}{l}\text { Total } \\
\text { Column }\end{array}$ & $\mathbf{8 . 8 5 2}$ & $\mathbf{5 . 3 8 5}$ & $\mathbf{8 . 0 3 4}$ & $\mathbf{4 . 5 4 2}$ & $\mathbf{2 . 9 7 6}$ \\
\hline Source: & Kristiyanti \& Pangemanan, 2020) &
\end{tabular}

Source: (Kristiyanti \& Pangemanan, 2020)

Table 2 below is the result of normalizing the comparison matrix in pairs based on the criteria:
Table 2. Normalizing Matrix Based on Criteria

Normalizing Comparison Matrix in Pairs Criteria

\begin{tabular}{|c|c|c|c|c|c|c|c|}
\hline Criteria & $\frac{\vec{\Xi}}{\underline{J}}$ & 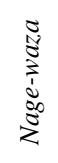 & 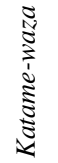 & 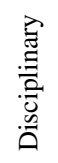 & 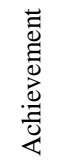 & 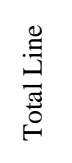 & 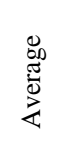 \\
\hline Ukemi & 0.113 & 0.071 & 0.099 & 0.121 & 0.156 & 0.560 & 0.112 \\
\hline Nage-waza & 0.296 & 0.186 & 0.259 & 0.153 & 0.162 & 1.055 & 0.211 \\
\hline \multicolumn{8}{|l|}{ Katame- } \\
\hline waza & 0.142 & 0.089 & 0.124 & 0.106 & 0.162 & 0.623 & 0.125 \\
\hline Disciplinary & 0.205 & 0.268 & 0.259 & 0.220 & 0.185 & 1.137 & 0.227 \\
\hline Achievement & 0.243 & 0.386 & 0.259 & 0.400 & 0.336 & 1.625 & 0.325 \\
\hline & & & & & & $\begin{array}{r}\text { Eigen } \\
\text { Vector }\end{array}$ & 1.000 \\
\hline
\end{tabular}

\section{Based on Self-Dropping Technique (Ukemi)}

The comparison matrix in pairs based on the Self-Dropping Technique (Ukemi) from the results of the processed questionnaire data produces the following tables 3:

Table 3. Matrix Self-Dropping Technique (Ukemi)

\begin{tabular}{lcccc}
\hline \multicolumn{4}{c}{ Comparison Matrix in Pairs Criteria } & \\
\hline & $\begin{array}{c}\text { Fajar } \\
\text { Maulana }\end{array}$ & $\begin{array}{c}\text { Muhammad } \\
\text { Vernanda }\end{array}$ & $\begin{array}{c}\text { Muhammad } \\
\text { Reza Dwi } \\
\text { Kurnia }\end{array}$ & Adhithana \\
\hline Fajar Maulana & 1.000 & 8.320 & 5.313 & 5.000 \\
\hline $\begin{array}{l}\text { Muhammad } \\
\text { Vernanda }\end{array}$ & 0.120 & 1.000 & 0.215 & 0.232 \\
\hline $\begin{array}{l}\text { Muhammad Reza } \\
\text { Dwi Kurnia }\end{array}$ & 0.188 & 4.642 & 1.000 & 0.693 \\
\hline Adhithana & 0.200 & 3.915 & 1.260 & 1.000 \\
\hline Total Column & $\mathbf{1 . 5 0 8}$ & $\mathbf{1 7 . 8 7 7}$ & $\mathbf{7 . 7 8 9}$ & $\mathbf{6 . 9 2 5}$
\end{tabular}

Source: (Kristiyanti \& Pangemanan, 2020)

Table 4 below are the results of the calculation of the normalization of comparison matrix in pairs based on the criteria of the Dropping Technique (Ukemi).

Table 4. Normalization Matrix of Self-Dropping Technigue (Ukemi) Comparison Matrix in Pairs Criteria

\begin{tabular}{|c|c|c|c|c|c|c|}
\hline & 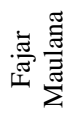 & 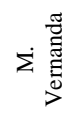 & 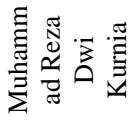 & 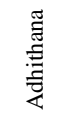 & $\begin{array}{l}\text { Total } \\
\text { Line }\end{array}$ & $\begin{array}{c}\text { Average } \\
\text { Value }\end{array}$ \\
\hline $\begin{array}{l}\text { Fajar } \\
\text { Maulana } \\
\end{array}$ & 0.663 & 0.465 & 0.682 & 0.722 & 2.533 & 0.633 \\
\hline $\begin{array}{l}\text { Muhammad } \\
\text { Vernanda }\end{array}$ & 0.080 & 0.056 & 0.028 & 0.034 & 0.197 & 0.049 \\
\hline $\begin{array}{l}\text { Muhammad } \\
\text { Reza Dwi } \\
\text { Kurnia }\end{array}$ & 0.125 & 0.260 & 0.128 & 0.100 & 0.613 & 0.153 \\
\hline Adhithana & 0.133 & 0.219 & 0.162 & 0.144 & 0.658 & 0.164 \\
\hline & & & & & $\begin{array}{l}\text { Eigen } \\
\text { Vector }\end{array}$ & 1.000 \\
\hline
\end{tabular}

\section{Based on Slamming Technique (Nage- waza)}


Comparison matrix in pairs based on the Slamming Technique (Nage-waza) from the results of the processed questionnaire data produces the following table 5 :

Table 5. Slamming Technigue Matrix (Nage-waza)

\begin{tabular}{lrrrr}
\hline \multicolumn{5}{c}{ Comparison Matrix in Pairs Criteria } \\
\hline & $\begin{array}{c}\text { Fajar } \\
\text { Maulana }\end{array}$ & $\begin{array}{c}\text { M. } \\
\text { Vernanda }\end{array}$ & $\begin{array}{c}\text { M. Reza } \\
\text { Dwi } \\
\text { Kurnia }\end{array}$ & Adhithana \\
\hline Fajar Maulana & 1.000 & 9.000 & 7.000 & 6.542 \\
\hline M. Vernanda & 0.111 & 1.000 & 0.200 & 0.255 \\
\hline $\begin{array}{l}\text { M.Reza Dwi } \\
\text { Kurnia }\end{array}$ & 0.143 & 5.000 & 1.000 & 1.000 \\
\hline Adhithana & 0.153 & 3.915 & 1.000 & 1.000 \\
\hline Total Column & $\mathbf{1 . 4 0 7}$ & $\mathbf{1 8 . 9 1 5}$ & $\mathbf{9 . 2 0 0}$ & $\mathbf{8 . 7 9 8}$ \\
\hline Source: (Kristiyant
\end{tabular}

Source: (Kristiyanti \& Pangemanan, 2020)

Table 6 below are the results of the calculation of the normalization of the comparison matrix in pairs based on the Slamming Technique (Nage-waza):

Table 6. Normalization Matrix of Slamming Technigue (Nage-waza)

\begin{tabular}{|c|c|c|c|c|c|c|}
\hline \multicolumn{7}{|c|}{ Comparison Matrix in Paris Criteria } \\
\hline & 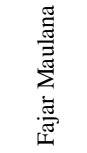 & 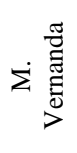 & 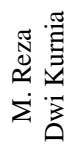 & 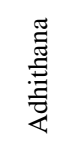 & 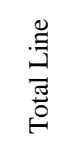 & 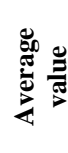 \\
\hline $\begin{array}{l}\text { Fajar } \\
\text { Maulana }\end{array}$ & 0.711 & 0.476 & 0.761 & 0.744 & 2.691 & 0.673 \\
\hline $\begin{array}{l}\text { M. } \\
\text { Vernanda }\end{array}$ & 0.079 & 0.053 & 0.022 & 0.029 & 0.183 & 0.046 \\
\hline $\begin{array}{l}\text { M. Reza } \\
\text { Dwi Kurnia }\end{array}$ & 0.102 & 0.264 & 0.109 & 0.114 & 0.588 & 0.147 \\
\hline Adhithana & 0.109 & 0.207 & 0.109 & 0.114 & 0.538 & 0.134 \\
\hline
\end{tabular}

Source: (Kristiyanti \& Pangemanan, 2020)

4. Based on the Lockdown or Laying Technique Criteria (Katame-waza)

The comparison matrix in pairs based on the Lockdown/Laying Technique (Katame-waza) from the questionnaire result data produces the following table 7 :

Table 7. Lockdown or Laying Technique Matrix (Katame-waza)

\begin{tabular}{lcccc}
\hline \multicolumn{5}{c}{ Comparison Matrix in Pairs Criteria } \\
\hline & $\begin{array}{c}\text { Fajar } \\
\text { Maulana }\end{array}$ & $\begin{array}{c}\text { M. } \\
\text { Vernanda }\end{array}$ & $\begin{array}{c}\text { M. Reza } \\
\text { Dwi } \\
\text { Kurnia }\end{array}$ & Adhithana \\
\hline Fajar Maulana & 1.000 & 5.646 & 3.000 & 3.000 \\
\hline $\begin{array}{l}\text { Muhammad } \\
\text { Vernanda }\end{array}$ & 0.177 & 1.000 & 0.347 & 0.333 \\
\hline $\begin{array}{l}\text { Muhammad } \\
\text { Reza Dwi } \\
\text { Kurnia }\end{array}$ & 0.333 & 2.884 & 1.000 & 0.794 \\
\hline Adhithana & 0.333 & 3.000 & 1.260 & 1.000 \\
\hline \multicolumn{1}{c}{ Total Colom } & $\mathbf{1 . 8 4 4}$ & $\mathbf{1 2 . 5 3 1}$ & $\mathbf{5 . 6 0 7}$ & $\mathbf{5 . 1 2 7}$ \\
\hline
\end{tabular}

Source: (Kristiyanti \& Pangemanan, 2020)
Table 8 below are the results of the calculation of the normalization of the comparison matrix in pairs based on the Lockdown /Lying Technique (Katame-waza):

Table 8. Normalization of the Communication Matrix

\begin{tabular}{lcccccc}
\hline \multicolumn{7}{c}{ Comparison Matrix in Pairs Criteria } \\
\hline & $\begin{array}{c}\text { Fajar } \\
\text { Maulana }\end{array}$ & $\begin{array}{c}\text { M. } \\
\text { Vernanda }\end{array}$ & $\begin{array}{c}\text { M. Reza } \\
\text { Dwi } \\
\text { Kurnia }\end{array}$ & Adhithana & $\begin{array}{c}\text { Total } \\
\text { Line }\end{array}$ & $\begin{array}{c}\text { Average } \\
\text { Value }\end{array}$ \\
\hline $\begin{array}{l}\text { Fajar } \\
\text { Maulana }\end{array}$ & 0.542 & 0.451 & 0.535 & 0.585 & 2.113 & $\mathbf{0 . 5 2 8}$ \\
\hline $\begin{array}{l}\text { M. } \\
\text { Vernanda }\end{array}$ & 0.096 & 0.080 & 0.062 & 0.065 & 0.303 & $\mathbf{0 . 0 7 6}$ \\
\hline $\begin{array}{l}\text { M. Reza } \\
\text { Dwi } \\
\text { Kurnia }\end{array}$ & 0.181 & 0.230 & 0.178 & 0.155 & 0.744 & $\mathbf{0 . 1 8 6}$ \\
\hline Adhithana & 0.181 & 0.239 & 0.225 & 0.195 & 0.840 & $\mathbf{0 . 2 1 0}$ \\
\hline & & \multicolumn{4}{c}{ EigenVector } & $\mathbf{1 . 0 0 0}$ \\
\hline
\end{tabular}

\section{Based on Disciplinary}

The comparison matrix in pairs based on Discipline from the processed questionnaire data results in the following table 9:

Table 9. Discplinary Matrix

\begin{tabular}{|c|c|c|c|c|}
\hline \multicolumn{5}{|c|}{ Comparison Matrix in Pairs Criteria } \\
\hline & $\begin{array}{c}\text { Fajar } \\
\text { Maulana }\end{array}$ & $\begin{array}{c}\text { M. } \\
\text { Vernanda }\end{array}$ & $\begin{array}{c}\text { M. Reza } \\
\text { Dwi Kurnia }\end{array}$ & Adhithana \\
\hline $\begin{array}{l}\text { Fajar } \\
\text { Maulana }\end{array}$ & 1.000 & 1.077 & 0.693 & 0.200 \\
\hline M. Vernanda & 0.928 & 1.000 & 0.500 & 0.188 \\
\hline $\begin{array}{l}\text { M. Reza Dwi } \\
\text { Kurnia }\end{array}$ & 1.442 & 2.000 & 1.000 & 0.255 \\
\hline Adhithana & 5.000 & 5.313 & 3.915 & 1.000 \\
\hline Total Kolom & 8.371 & 9.391 & 6.108 & 1.644 \\
\hline
\end{tabular}

Tabel 10 below are the results of the calculation of the normalization of comparison matrix in pairs based on Disciplin:

Tabel 10. Normalization of Discipline Matrix

\begin{tabular}{|c|c|c|c|c|c|c|}
\hline \multicolumn{7}{|c|}{ Comparison Matrix in Pairs Criteria } \\
\hline & 点 $\frac{\tilde{\Xi}}{\tilde{\Xi}}$ & 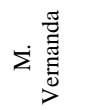 & 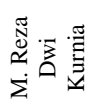 & 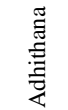 & 营 & 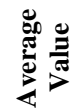 \\
\hline $\begin{array}{l}\text { Fajar } \\
\text { Maulana }\end{array}$ & 0.119 & 0.115 & 0.114 & 0.122 & 0.469 & 0.117 \\
\hline M.Vernanda & 0.111 & 0.106 & 0.082 & 0.115 & 0.414 & 0.103 \\
\hline $\begin{array}{l}\text { M. Reza } \\
\text { Dwi } \\
\text { Kurnia }\end{array}$ & 0.172 & 0.213 & 0.164 & 0.155 & 0.704 & 0.176 \\
\hline Adhithana & 0.597 & 0.566 & 0.641 & 0.608 & 2.412 & 0.603 \\
\hline \multicolumn{6}{|c|}{ Eigen Vector } & 1.000 \\
\hline
\end{tabular}

\section{Based on Achievement}


The comparison matrix in pairs based on Achievement from the results of the processed questionnaire data produce the following table 11.

Table 11. Achievement Criteria Matrix

\begin{tabular}{ccccc}
\hline \multicolumn{5}{c}{ Comparison Matrix in Pairs Criteria } \\
\hline & $\begin{array}{c}\text { Fajar } \\
\text { Maulana }\end{array}$ & $\begin{array}{c}\text { Muhammad } \\
\text { Vernanda }\end{array}$ & $\begin{array}{c}\text { M Reza } \\
\text { Dwi } \\
\text { Kurnia }\end{array}$ & Adhithana \\
\hline $\begin{array}{c}\text { Fajar } \\
\text { Maulana }\end{array}$ & 1.000 & 8.653 & 6.649 & 5.944 \\
\hline $\begin{array}{c}\text { Muhammad } \\
\text { Vernanda }\end{array}$ & 0.116 & 1.000 & 0.250 & 0.250 \\
\hline $\begin{array}{c}\text { Muhammad } \\
\text { Reza Dwi } \\
\text { Kurnia }\end{array}$ & 0.150 & 4.000 & 1.000 & 1.000 \\
\hline Adhithana & 0.168 & 4.000 & 1.000 & 1.000 \\
\hline $\begin{array}{c}\text { Total } \\
\text { Column }\end{array}$ & $\mathbf{1 . 4 3 4}$ & $\mathbf{1 7 . 6 5 3}$ & $\mathbf{8 . 8 9 9}$ & $\mathbf{8 . 1 9 4}$ \\
\hline Source: (Kristiyanti \& Pangemanan, 2020) &
\end{tabular}

Table 12 below are the results of the calculation of the normalization of comparison matrix in pairs based on Achievement.

Table 12. Normalization of Achievement Matrix

\begin{tabular}{|c|c|c|c|c|c|c|}
\hline \multicolumn{7}{|c|}{ Comparison Matrix in Pairs Criteria } \\
\hline & 㺃㺃 & 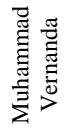 & 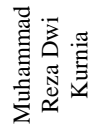 & 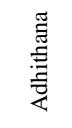 & 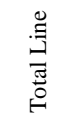 & $\begin{array}{c}\text { Aver } \\
\text { age } \\
\text { Value }\end{array}$ \\
\hline $\begin{array}{l}\text { Fajar } \\
\text { Maulana }\end{array}$ & 0.697 & 0.490 & 0.747 & 0.725 & 2.660 & 0.665 \\
\hline $\begin{array}{l}\text { Muhammad } \\
\text { Vernanda }\end{array}$ & 0.081 & 0.057 & 0.028 & 0.031 & 0.196 & 0.049 \\
\hline $\begin{array}{l}\text { Muhammad } \\
\text { Reza Dwi } \\
\text { Kurnia }\end{array}$ & 0.105 & 0.227 & 0.112 & 0.122 & 0.566 & 0.141 \\
\hline Adhithana & 0.117 & 0.227 & 0.112 & $\frac{0.122}{\mathbf{E i}}$ & $\begin{array}{ll}0.578 \\
\text { Vektor }\end{array}$ & 0.145 \\
\hline
\end{tabular}

Source:(Kristiyanti \& Pangemanan, 2020)

\section{Combined Multiplication}

Combined multiplication is the multiplication between the average value of the criteria determining the best Judo athlete and the average value of the alternative determining the best Judo athlete.

$$
\begin{aligned}
& \text { Fajar Maulana } \\
& \text { Muhammad Vernanda } \\
& \text { Muhammad Reza Dwi Kurnia } \\
& \text { Adhithana } \\
& =\left[\begin{array}{lllll}
0,633 & 0,673 & 0,528 & 0,117 & 0,665 \\
0,049 & 0,046 & 0,076 & 0,103 & 0,049 \\
0,153 & 0,147 & 0,186 & 0,176 & 0,141 \\
0,164 & 0,134 & 0,210 & 0,603 & 0,145
\end{array}\right] \times\left[\begin{array}{l}
0,112 \\
0,211 \\
0,125 \\
0,227 \\
0,325
\end{array}\right] \\
& =\left[\begin{array}{l}
0,521 \\
0,064 \\
0,157 \\
0,257
\end{array}\right]
\end{aligned}
$$

From the result of the combined multiplication above shows that in determining the best Judo athletes are Fajar Maulana with value 0.521 then Adhithana with value 0.257, Muhammad Reza Dwi Kurnia with value of 0.157 and Muhammad Vernanda with value 0.064 . Measuring consistency of $\mathrm{M}$ and M with:

$\mathrm{M}=\mathrm{CI}$ level-2 + (vector eigen level-2) (CI level-3) dan

Ḿ = RI level-2 + (vector eigen level-2) (RI level-3).

The calculation to measure the consistency of the employee matrix is based on the following:

$$
\begin{aligned}
& M=0,029+\left[\begin{array}{lllll}
0,112 & 0,211 & 0,125 & 0,227 & 0,325
\end{array}\right] \times\left[\begin{array}{l}
0,029 \\
0,066 \\
0,010 \\
0,005 \\
0,050
\end{array}\right. \\
& =0,029+\left[\begin{array}{lllll}
0,003 & 0,014 & 0,001 & 0,001 & 0,016
\end{array}\right] \\
& =0,029+0,036 \\
& =0,064 \\
& \dot{\mathrm{M}}=1,120+\left[\begin{array}{lllll}
0,112 & 0,211 & 0,125 & 0,227 & 0,325
\end{array}\right] \quad \mathrm{X}\left[\begin{array}{l}
1,120 \\
1,120 \\
1,120 \\
1,120 \\
1,120
\end{array}\right] \\
& =1,120+\left[\begin{array}{lllll}
0,125 & 0,236 & 0,140 & 0,255 & 0,364
\end{array}\right] \\
& =1,120+1,120 \\
& =2,240
\end{aligned}
$$

Calculation HCR (Hierarchy Consistency Ratio)

$$
\begin{aligned}
& \mathrm{CRH}=\mathrm{M} / \overline{\mathrm{M}} \\
& =0,036 / 2,240 \\
& =0,03
\end{aligned}
$$

\section{Data Calculation Using Expert Choice 2000}

After calculating the data using the Analytical Hierarchy Process (AHP) method manually, data calculation will be performed using the Expert Choice 2000 Software as follows:

\section{a. Based on Criteria}

Here are the input values of comparison matrix in pairs based on criteria.

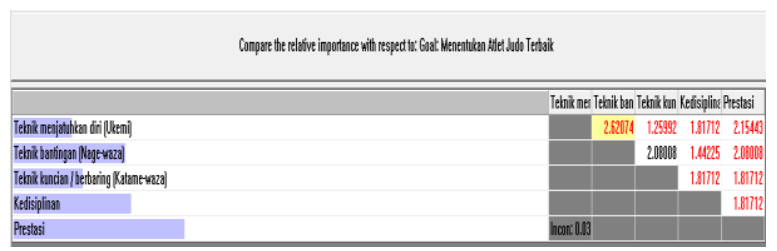

Source: (Kristiyanti \& Pangemanan, 2020)

Figure 2. Matrix Input Based on Criteria

Figure 3 below is a vector of eigenvectors based on criteria

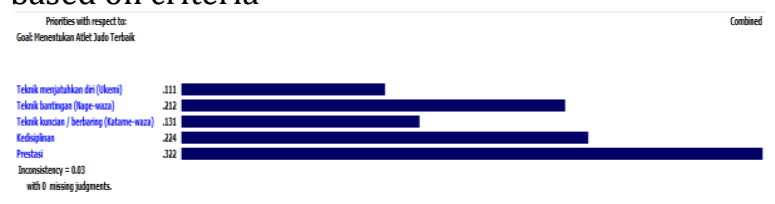

Source: (Kristiyanti \& Pangemanan, 2020)

Figure 3. Graph of Matrix Input Results Based on Criteria

Based on the graph above shows that Achievement is the highest criterion in the best Judo athletes with a value of 0.322 , then Discipline with a value of 0.224 , a Dage Technique (Nagewaza) with a value of 0.212, a Locking / Lying Technique (Katame-waza) with a value of 0.131 and a Technique Dropping Down (Ukemi) with a value of 0.111 . 
b. Self-Dropping Technique Criteria (Ukemi)

The following is a value input of comparison matrix in pair based on the criteria of the SelfDropping Technique (Ukemi).

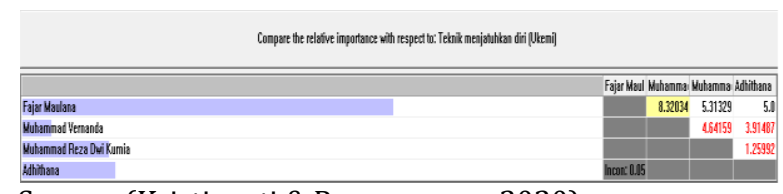

Source: (Kristiyanti \& Pangemanan, 2020)

Figure 4. Matrix Dropping Technique Input (Ukemi)

Figure 5 below this is a vector eigen graph based on the criteria for the Self-Dropping Technique (Ukemi).

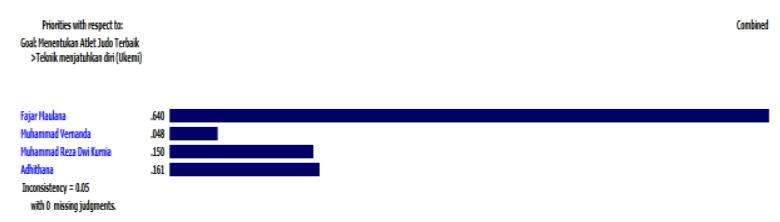

Source: (Kristiyanti \& Pangemanan, 2020)

Figure 5. Result Graph of Creteria Matrix Input

Based on the graph above shows that in determining the best Judo athletes with the criteria of the Self-Dropping Technique (Ukemi), Fajar Maulana took first place with value of 0.640 , then Adhithana with value 0.161, Muhammad Reza Dwi Kurnia with value 1501 and Muhammad Vernanda with value 0.048 .

c. Criteria of Slamming Technique (Nage-waza)

The following are input values for comparison matrix in pairs based on Slamming Technique criteria (Nage-waza).

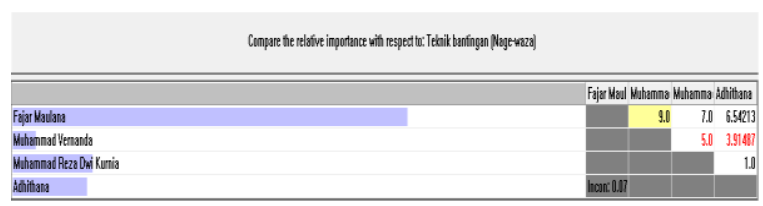

Source: (Kristiyanti \& Pangemanan, 2020)

Figure 6. Matrix Input of Technical Criteria (Nagewaza)

Figure 7 below is a vector eigenvector based on the Nage-waza technique criteria.

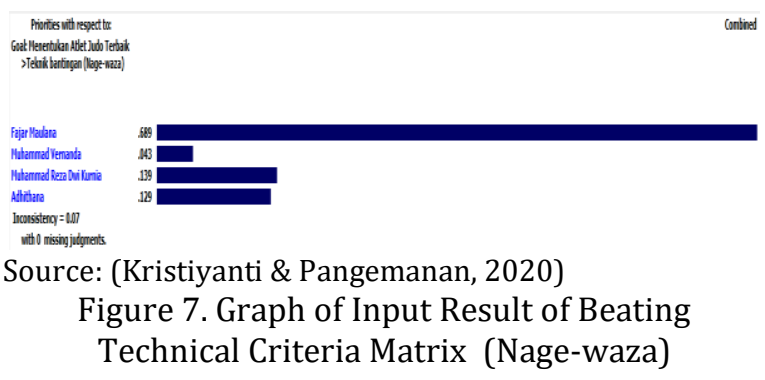

Based on the graph above shows that in the selection of achievers with the criteria of Beating Technique (Nage-waza), Fajar Maulana took first place with a value of 0.689 , then Reza Dwi Kurnia with a value of 0.139 , Adhithana with a value of 0.129 and Muhammad Vernanda with a value of 0.043 .

d. Lockdown/Laying Technique Criteria (Katame-waza)

The following are input values of comparison matrix in pairs based on the Lockdown/Laying Technique (Katame-waza) criteria.

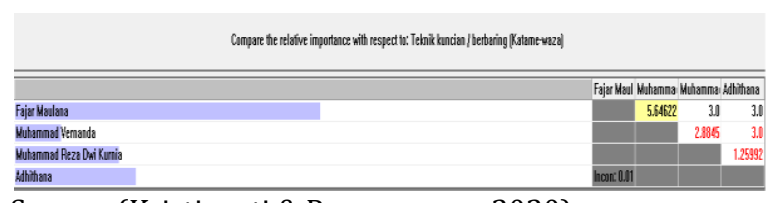

Source: (Kristiyanti \& Pangemanan, 2020)

Figure 8. Locking Technique Matrix Input / Lying Criteria (Katame-waza)

Figure 9 below is a vector eigen graph based on the Lockdown/Laying Technique criteria (Katame-waza).

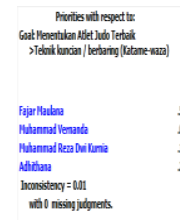

Figure 9. Result Graph of Lock/Lay Matrix Input Criteria (Katame-waza)

Based on the chart above shows that in determining the best Judo athletes with the criteria of Lock / Lay Technique (Katame-waza), Fajar Maulana took first place with a value of 0.530 , then Adhithana with a value of 0.210 , Muhammad Reza Dwi Kurnia with a value of 0.185 and Muhammad Vernanda with a value of 0.075 .

\section{e. Disciplinary Criteria}

The following is value input of comparison matrix in pairs based on Disciplinary criteria.

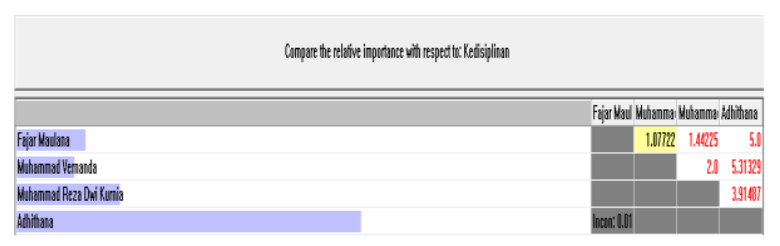

Source: (Kristiyanti \& Pangemanan, 2020)

Figure 10. Disciplinary Criteria Matrix Input

Figure 11 below is a vector eigen graph based on disciplinary criteria. 


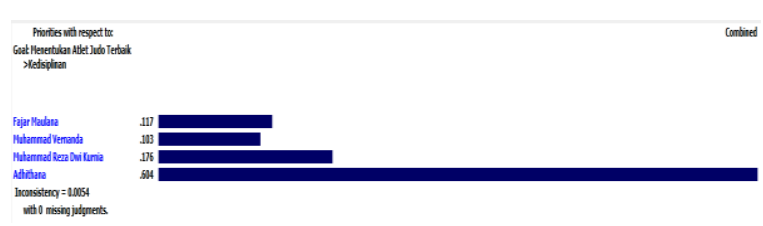

Source:(Kristiyanti \& Pangemanan, 2020)

Figure 11. Result Graph of Disciplinary Criteria Matrix Input

Based on the graph figure 11 above shows that in determining the best Judo athletes with Disciplinary criteria, Adhithana took first place with a value of 0.604 , then Muhammad Reza Dwi Kurnia with a value of 0.176 , Fajar Maulana with a value of 0.117 and Muhammad Vernanda with a value of 0.103 .

\section{f. Achievement}

The following is input of comparison matrix value in pairs based on the Achievement criteria.

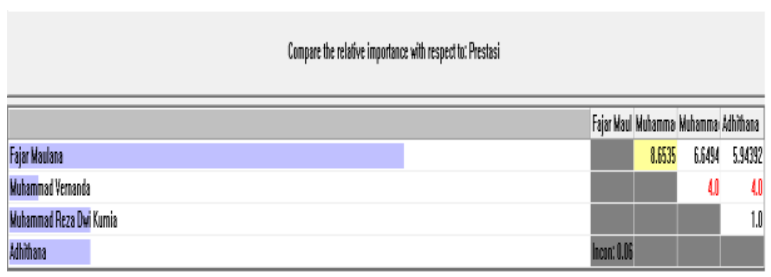

Source: (Kristiyanti \& Pangemanan, 2020)

Figure 12. Input of Achievement Criteria Matrix

Figure 13 below is a vector eigen graph based on the Achievement criteria.

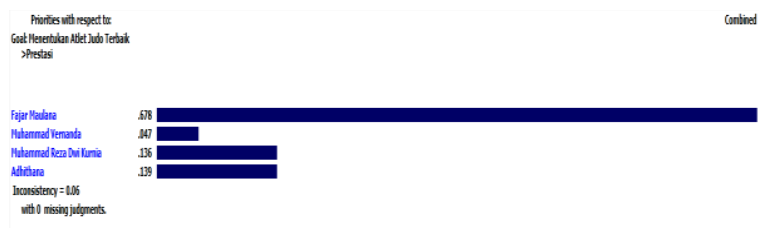

Source: (Kristiyanti \& Pangemanan, 2020)

Figure 13. Graph of Input Result of Achievement Criteria Matrix

Based on the graph figure 13 above shows that in determining the best Judo athlete with Achievement criteria, Fajar Maulana took first place with a value of 0.678 , then Adhithana with a value of 0.139 , Muhammad Reza Dwi Kurnia with a value of 0.136 and Muhammad Vernanda with a value of 0.047 .

g. The final result of calculating expert choice 2000 software

Figure 14 below is the final result of the calculation of expert choice 2000 software to determine the best Judo athlete:

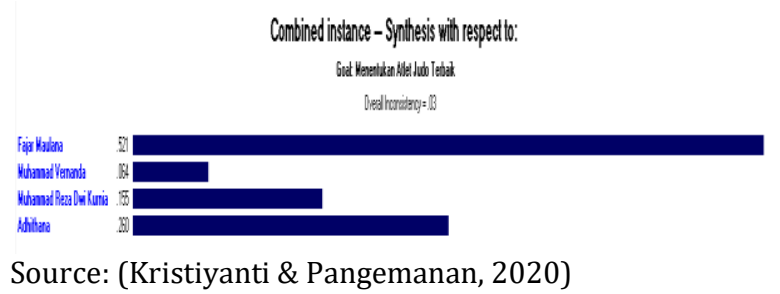

Figure 14. Graph Synthesize With Respect To Goal

Based on the synthesize graph with respect to the goal above figure 14 in determining the best Judo athlete with the criteria of Dropping Technique (Ukemi), Dings Technique (Nage-waza), Locking / Lying Technique (Katame-waza), Discipline and Achievement are Fajar Maulana occupying the first position with a value of 0.521 then Adhithana with a value of 0.260, Muhammad Reza Dwi Kurnia with a value of 0.155 and Muhammad Vernanda with a value of 0.064 , and the obtained value of Overall Inconsistency or $\mathrm{CRH}$ 0.03 .

\section{CONCLUSION}

Based on the purpose of this research, data processing and analysis that has been done by the author, the conclusions are drawn based on the calculation of the Analytical Hierarchy Process (AHP) in which obtained priority criteria that are most important in determining the best Judo athletes where Achievement criteria with a value of 0.325 or equivalent to $32 \%$, then further followed by Disciplinary criteria with a value of 0.227 or equivalent to 23\%, a criterion of Damping Technique (Nage-waza) with a value of 0.211 or equivalent to 21\%, Lockout/Lying Technique Criteria (Katame-waza) with a value of 0.125 or equivalent to $12 \%$ and the last ranking is the criteria for the Self-Dropping Technique (Ukemi) with a value of 0.112 or equivalent to $11 \%$. The analysis result of the Analytical Hierarchy Process calculation stated that in determining the best Judo athlete was Fajar Maulana. AHP calculation data obtained from 3 questionnaires filled out by respondents and the final result obtained that Fajar Maulana is superior to 0.521 or $52.15 \%$. While the second priority is owned by Adhithana with a value of 0.257 or $25.71 \%$ the third priority is owned by Muhammad Reza Dwi Kurnia with a value of 0.157 or $15.74 \%$ and the last priority is owned by Muhammad Vernanda with a value of 0.064 or $6.40 \%$.

\section{REFERENCES}

Agung, H., \& Ricky. (2016). Aplikasi Sistem Pendukung Keputusan Untuk Pemilihan Siswa Teladan Menggunakan Metode Topsis. 
Jurnal Ilmiah FIFO, 8(2), 112-126. https://doi.org/10.22441/fifo.v8i2.1306

Ariani, F. (2017). Sistem Penunjang Dalam Penentuan Prioritas Pemilihan Percetakan Media Promosi Menggunakan Metode AHP. Jurnal Informatika, 4(2), 214-221.

Desi Ratna, Windarto, A. P., \& Hartama, D. (2018). Sistem Pendukung Keputusan untuk Rekomendasi Kelulusan Sidang Menggunakan Metode AHP-TOPSIS. 6(November 2017), 1-6. https://doi.org/10.14710/jtsiskom.6.1.2018. 1-6

Handayani, R. I., \& Marzuki, A. (2018). Sistem Pendukung Keputusan Penerimaan Karyawan Dengan Menggunakan Analytical Hierarchy Process (AHP) Studi Kasus: PT. Virtus Venturama. Pilar Nusa Mandiri, 14(1), 43. https://doi.org/10.1007/s11276-0066154-9

Herman Firdaus, I., Abdillah, G., \& Renaldi, F. (2016). Sistem Pendukung Keputusan Penentuan Karyawan Terbaik Menggunakan Metode AHP Dan Topsis. Seminar Nasional Teknologi Informasi Dan Komunikasi, 2016(Sentika), 2089-9815.

Imandasari, T., Wanto, A., \& Windarto, A. P. (2018). Analisis Pengambilan Keputusan dalam Menentukan Mahasiswa PKL Menggunakan Metode Promethee. Jurnal Riset Komputer (JURIKOM), 5(3), 234-239.

Kristiyanti, D. A. (2017). Sistem Pendukung Keputusan Seleksi Karyawan Untuk Jabatan Tertentu Dengan Pendekatan Analisa Gap Profile Matching. Paradigma - Jurnal Komputer Dan Informatika, 19(1), 20-29. https://doi.org/10.31294/P.V19I1.1502

Kristiyanti, D. A., \& Pangemanan, G. W. (2020). Laporan Akhir Penelitian: Sistem Pendukung Keputusan Dalam Menentukan Atlet Judo Terbaik Menggunakan Metode Ahp.

Kurniawan, W. J., \& Gusrianty. (2018). SISTEM PENDUKUNG KEPUTUSAN SELEKSI ATLET POOMSAE TAEKWONDO DENGAN METODE ANALITYC HIERACHY PROCESS. JOISIE Journal Of Information System And Informatics Engineering, 2(1), 26-32.

Mardiati, K. S., \& Oktafianto. (2017). Sistem Pendukung Keputusan (DSS) Penerima Bantuan Rumah Tak Layak Huni (RTLH) Pada Kecamatan Ambarawa Dengan Menggunakan Metode Analytical Hierarcy Process (AHP). Appl. Phys. A, 73, 1-21.

Nadeak, B., Atun, S. A., \& Fadlina. (2018). Sistem Pendukung Keputusan Dalam Menentukanemain Basket Terbaik Menggunakan Algoritma Analytical Hierarchy
Process ( AHP ) ( Study Kasus Klub Angsapura Sania Medan ). JURIKOM (Jurnal Riset Komputer), 5(2), 193-207.

Nurdiyanto, H., \& Meilia, H. (2016). Sistem Pendukung Keputusan Penentuan Prioritas Pengembangan Industri Kecil Dan Menengah Di Lampung Tengah Menggunakan Analitical Hierarchy Process (Ahp). In S. A. Yogyakarta (Ed.), Semnasteknomedia Online (Vol. 4, Issue 1, pp. 3.3-37-3.3-42). Seminar Nasional Teknologi Informasi dan Multimedia 2016. https://ojs.amikom.ac.id/index.php/semnast eknomedia/article/view/1163

Polmetra, Nurcahyo, G. W., \& Defit, S. (2015). Sistem Pendukung Keputusan Dengan Metode Analitical Hierarchy Procces (Ahp) Untuk Pemilihan Pelatih Bulutangkis. In U. P. I. Y. Padang (Ed.), Sistem Pendukung Keputusan Dengan Metode Analitical Hierarchy Procces (Ahp) Untuk Pemilihan Pelatih Bulutangkis (Vol. 1, Issue Senatkom, pp. 131-137). Prosiding Seminar Ilmiah Nasional Teknologi Komputer (SENATKOM 2015).

Ruskan, E. L., Ibrahim, A., \& Hartini, D. C. (2013). Sistem Pendukung Keputusan Pemilihan Hotel Di Kota Palembang Dengan Metode Simple Additive Weighting (Saw). Jurnal Sistem Informasi, 5(1), 546-565.

Saefudin, \& Wahyuningsih, S. (2014). Sistem Penunjang Keputusan Penilaian Kinerja Pegawai Menggunakan Metode Analytical Hierarchy Process (AHP) Pada RSUD Serang. Jurnal Sistem Informasi, 1(1), 33-37. https://doi.org/10.36723/juri.v9i2.107

Sanyoto, G. P., Handayani, R. I., \& Widanengsih, E. (2017). Sistem Pendukung Keputusan Pemilihan Laptop Untuk Kebutuhan Operasional Dengan Metode Ahp ( Studi Kasus : Jurnal Pilar Nusa Mandiri Vol.13, No. 2., 13(2), 167-174. https://doi.org/ISSN: 2303-11

Stevanus, R., Handayani, R. I., \& Kristiyanti, D. A. (2017). Sistem Pendukung Keputusan Pemberian Bonus Karyawan Menggunakan Metode Ahp Pada Rumah Sakit Buah Hati Ciputat. Sistem Pendukung Keputusan Pemberian Bonus Karyawan Menggunakan Metode Ahp Pada Rumah Sakit Buah Hati $\begin{array}{lll}\text { Ciputat, } & \text { 2(2), }\end{array}$ http://jurnal.una.ac.id/index.php/jurti/articl e/view/42/39

Sundari, D. S. (2018). Perubahan Fungsi Judo Pada Masyarakat Jepang. Universitas Sumatera Utara. 